\title{
A Comparative Study of Ultrasonography and Contrast Enhanced Computed Tomography in Acute Abdominal Trauma
}

\author{
Ballari Sanjay ${ }^{1}$, C. Sanjeevi ${ }^{2}$ \\ ${ }^{1}$ Resident, Department of Radiodiagnosis, SSSMCRI, Chennai, Tamilnadu, ${ }^{2}$ Associate Professor, Department of Radiodiagnosis, \\ SSSMCRI, Chennai, Tamilnadu, India
}

Corresponding author: C. Sanjeevi, Associate Professor, Department of Radiodiagnosis, SSSMCRI, Chennai, Tamilnadu, India

DOI: http://dx.doi.org/10.21276/ijcmsr.2020.5.2.11

How to cite this article: Ballari Sanjay, C. Sanjeevi. A comparative study of ultrasonography and contrast enhanced computed tomography in acute abdominal trauma. International Journal of Contemporary Medicine Surgery and Radiology. 2020;5(2):B47-B51.

\section{A B S T R A C T}

Introduction: Ultrasonography for blunt abdominal trauma is the primary screening examination for blunt abdominal trauma in most trauma centers. CT is to define the site and extent of organ injuries, but also to exclude other significant injury, thereby avoiding unnecessary surgery. The objective of our study was to evaluate the role of US and CECT for the depiction of organ injury and compare the findings of these two frequently used modalities

Material and methods: This was a cross sectional study which included 40 patients with no age predilection with history of trauma referred to radiology department for US and CT for a period of 18 months (Jan 2018 - June 2019).

Results: Forty patients with a history of blunt abdominal trauma were evaluated by US and CT. There were 31 male and 9 female patients. The age of patients ranged from 8 to 75 years. The mean age was 37.85 years. 1 patient was child $(<12$ years). Eighteen patients were 40 years or older.

Conclusion: US is a valuable initial modality for evaluation of patients with abdominal trauma. CT is required in most US positive patients to delineate the exact extent of injury and exclude any other significant injuries. US or CT quantification of hemoperitoneum or grading of injury does not always dictate whether the management should be conservative or surgical. However they reflect the severity of injury and injuries of severe grade with large free fluid are more likely to require a laparotomy.

Keywords: US, CECT, Abdominal Trauma

\section{INTRODUCTION}

Trauma is also called as an 'unsolved epidemic' in the present society. Death from cancer and cardiovascular disease together is almost equal to loss of life from trauma. Trauma is the common cause of death in people less than 40 years $^{1,2}$ of age. Traditionally, abdominal injuries can be divided into penetrating trauma and blunt trauma. Blunt abdominal trauma (BAT) usually occurs due to road traffic accidents (RTA), fall from heights or during sports. ${ }^{2}$ Blunt abdominal trauma may represent an immediate threat to life and requires rapid diagnosis and treatment. In the remaining patients, in whom there is no immediate threat to life, a correct diagnosis is paramount in the interest of timely institution of appropriate therapy. 3,4

A diagnostic tool is required for assessment of abdominal injuries since clinical examination and physical examination is unreliable in most of the cases..$^{5}$ Previously available diagnostic techniques included plain radiography, contrast studies, angiography and scintigraphy. Diagnostic laparotomy used to play a prominent role, so much so that the popular surgical aphorism was "never let the abdominal wall stand between you and the diagnosis". ${ }^{6}$ Most of the patients with abdominal injuries can be conservatively managed ${ }^{5}$ and laparotomy involves significant morbidity and mortality which has led to look for better alternatives. ${ }^{7}$

Diagnostic peritoneal lavage (DPL) introduced in 1965 by Root et $\mathrm{al}^{2}$ was cheap and easily available technique and could be done safely and quickly even in emergency setting. The major disadvantage of DPL is its limitation in evaluating retroperitoneal organs like kidneys and pancreas which has led to additional method for diagnosis. ${ }^{8}$

CT is however relatively insensitive to early detection of intestinal, mesenteric and pancreatic injury. The need to shift a potentially unstable out of the trauma care area, the time required to prepare the patient, and limited availability are its main disadvantages. ${ }^{9}$ In real life, most injured patients are initially evaluated at the closest hospital.

The variable scope, sensitivity, accuracy and advantages of these three extensively used modalities has given rise to their variable use in different parts of the world. ${ }^{10}$ Today US is the initial modality in blunt abdominal trauma in Europe and Japan, whereas CT is the initial modality of choice in stable patients in North American centres. ${ }^{11,12}$ The popularity of 'emergent abdominal sonography' or 'focussed assessment sonography in trauma' and its validation as to its effectiveness in several trauma centres has renewed its interest in other trauma centres as well, particularly in the current era of 
cost containment in health scenario. ${ }^{13-15}$ The objective of our study was to evaluate the role of US and CECT for the depiction of organ injury and compare the findings of these two frequently

used modalities so that their optimal use can be maximised.

\section{MATERIAL AND METHODS}

The Cross sectional study involved all patients with diagnosis of acute abdominal trauma Department of Radio diagnosis, Shri Sathya Sai Medical College and Research Institute, Kanchipuram district. The time, cause and mode of injury was obtained whenever possible.

Forty patients who were stable to undergo US and CT had positive findings were subjected for the study. Whenever possible, US preceded CT and the time gap between the two was kept to the minimum to make the studies comparable. US and CECT were done in all 40 patients. Patients having the tests interpreted as negative and who either did not require admission or who were discharged after a short observation without any further investigation, were not included. Diagnostic peritoneal tapping was performed in all the patients with free fluid to confirm the presence of hemoperitoneum.

\section{Ultrasonography}

US scans were performed on "MINDRAY DC 7" machine, with 2-5 and 5-10

Mhz curvilinear and linear probes. Particular attention was paid in assessing free fluid

in abdomen and evaluation of solid organs.

\section{CT Technique}

CT scans were performed on "GE 16 SLICE" helical sub second $(0.75 \mathrm{Sec})$ scanner capable of 50 secs, continuous spiral run. Images were reconstructed with $180^{\circ}$ linear interpolation reconstruction algorithm. Helical CT of the entire abdomen was done from the level of dome of diaphragm up to the inferior aspect of ischium.

\section{Contrast Material}

Routine oral contrast was not given to the patients. All patients received IV contrast material administered with an automated Medrad Power Injector after the initial pilot scan via a large-bore $(18-20 \mathrm{G})$ peripheral venous line. A total of $100 \mathrm{ml}, 60 \%$ nonionic iodinated contrast was given intravenously at a rate of $2-4 \mathrm{ml}$ per second. Scanning was initiated 70-90 secs after initiation of contrast infusion. Free fluid with attenuation value > 30 Hounsfield Units (HU) was labelled as hemoperitoneum. Follow up US or CT scans were obtained as dictated by the clinical course of the patients. Patients undergoing conservative management were clinically followed up. Surgical findings were noted from the operative notes in those undergoing laparotomy.

Injury to different organs was staged by organ injury scaling (OIS) system developed by Organ Injury Scaling Committee of the American Association for the Surgery of Trauma (AAST), Moore et al. ${ }^{16}$ (1995).

\section{Inclusion Criteria}

1. All patients with history of blunt abdominal trauma.

2. Abdominal penetrating injured patients.
3. Cases are included irrespective of age \& sex.

\section{Exclusion Criteria}

1. Patients in hypovolemic shock / hemmorhagic shock.

2. Associated spinal injuries.

3. Patients with head injury.

4. Pregnant women.

5. Patients with chronic renal disease.

\section{Sample Size}

40 sample size was selected based on average number of cases with abdominal trauma presenting to Radio diagnosis department of SSSMC\&RI, which is 3 cases/month in average and based on number of old cases that satisfying inclusive and exclusive criteria.

\section{STATISTICAL ANALYSIS}

Data entered in excel sheet statistical analysis done by SPSS 23 software using descriptive statistics.

\section{RESULTS}

Forty patients with a history of blunt abdominal trauma were evaluated by US and CT. There were 31 male and 9 female patients. The age of patients ranged from 8 years to 75 years. The mean age was 37.85 years. One patient was child $(<12$ years). Eighteen patients were 40 years or older (table-1). The organs injured are shown in able-2. Spleen was most affected.

\section{US and CT detection of liver injuries}

Of the 22 cases of liver injuries, six had grade III injury. The remaining six cases had grade I and ten grade II injury. All cases were managed conservatively \& Surgically. The calculated sensitivity, specificity and accuracy of US in detection of hepatic injuries was $84 \%$, $99 \%$ and $86 \%$ respectively

\section{Ultrasound and CT detection of splenic injuries}

Of the 17 splenic injuries, grade III injuries were seen in 6 patients. Two patients had grade I and five patients had grade II injury. Four patients had grade IV injury. Their outcome was analyzed. The calculated sensitivity, specificity and accuracy of US detection of splenic injuries was 61\%, 100\% and $65 \%$ respectively. One case (case no.15) was excluded from calculation as overlying subcutaneous emphysema prevented ultrasound evaluation.

\section{Ultrasound and CT detection of kidney injuries}

There were Three kidney injuries. Two patients had left kidney injury and one patients had right kidney injury. Their grading and outcome was analyzed. There were two grade I injuries. One patients had grade IV injury. All the patients were surgically managed. USG detected only 1 case of renal trauma where CT diagnosed all the 3 cases correctly in addition to accurately showing the extent of injury and all these cases were graded using organ injury scale.

\section{Ultrasound and CT detection of pancreatic injuries}

Two patients had pancreatic injuries. Pancreas could not be evaluated on US in either of the cases because of bowel gas. In both the cases hemoperitoneum was detected on US and CT. CT revealed an area of hypodensity at the junction of the head and tail of the pancreas with hematoma in the peripancreatic region in one case and $1.4 \mathrm{~cm}$ laceration invoving the tail of 


\begin{tabular}{|l|c|c|c|}
\hline Age group & No of patients & Sex & Percentage \\
\hline $0-12$ & 01 & $1 \mathrm{M}$ & $3.2 \%$ \\
\hline $13-20$ & 03 & $2 \mathrm{M} \mathrm{1F}$ & $9.6 \%$ \\
\hline $21-30$ & 06 & $5 \mathrm{M} \mathrm{1F}$ & $19.3 \%$ \\
\hline $31-40$ & 07 & $6 \mathrm{M} \mathrm{1F}$ & $22.5 \%$ \\
\hline $41-50$ & 08 & $6 \mathrm{M} \mathrm{2F}$ & $25.8 \%$ \\
\hline $51-60$ & 05 & $4 \mathrm{M} \mathrm{1F}$ & $16.1 \%$ \\
\hline $61-70$ & 01 & $1 \mathrm{M}$ & $3.2 \%$ \\
\hline \multicolumn{4}{|c|}{ Table-1: Age group and gender } \\
\hline
\end{tabular}

\begin{tabular}{|l|c|c|}
\hline Organ & No of patients & Percentage \\
\hline Spleen & 13 & 41.95 \\
\hline Liver & 17 & $54.8 \%$ \\
\hline Kidneys & 3 & $9.6 \%$ \\
\hline Bowel and mesentery & 5 & $16.1 \%$ \\
\hline Pancreas & 2 & $6.4 \%$ \\
\hline Urinary bladder & 1 & $3.2 \%$ \\
\hline Diaphragm Table-2: Organ injuries \\
\hline \multicolumn{2}{|c|}{$3.2 \%$} \\
\hline
\end{tabular}

pancreas in the other. Both cases recovered on conservative \& Surgical management.

\section{Bowel and Mesentery}

Of the total 5 patients, four patients had only bowel injury and one patient had isolated mesenteric injury. Pneumoperitoneum was present in 3 out of 5 cases of bowel injury. The sensitivity, specificity and accuracy of US were 20\%, 100\%, 42.8\% and CT was positive in all patients and showed an overall sensitivity, specificity and accuracy of $100 \%$ respectively. Their grading and outcome was analyzed.

\section{Pelvic fractures and retroperitoneal hematoma}

Six patients had pelvic fractures, one of them had an associated sizable retroperitoneal hematoma on CT.

\section{Bladder}

Extra peritoneal bladder injury was detected in one patient with an associated spleen and kidney injury.

\section{Diaphragm}

One patient had diaphragmatic injury. Diaphragm could not be evaluated on US in those case because of bowel gas. Hemoperitoneum was detected on both US and CT and it was surgically managed.

\section{Pneumoperitoneum}

Pneumoperitoneum was detected in 2 patients. Two patients showed the presence of air under diaphragm in plain erect abdominal radiograph. The sensitivity, specificity and accuracy of X-Ray were 33.3\%, 100\%, 60\%. All the patients had associated other organ injuries and were surgically managed.

Out of the total 40 patients, in 24 patients US and CT showed similar findings.

In 8 patients CT detected additional finding or provided additional information but did not change the management. In 8 patients CT was decisive for management or surgical planning. However in all of them US showed the presence of free fluid.

In 39 patients US showed either intra-abdominal free fluid or organ injury or both. In one patient US did not reveal any abnormality. Liver injury was later detected on CT.

- US had an overall sensitivity of $96 \%$, specificity of $100 \%$ and accuracy of $96 \%$.

- CT was positive (either for intra-abdominal free fluid or organ injury or both) in all the patients and thus showed an overall sensitivity, specificity and accuracy of $100 \%$.

CT showed distinct advantage in patients with overlying subcutaneous emphysema which prevented normal visualization of underlying structures on US (in case no.15). The patient had splenic injury. CT was useful in detecting associated injuries such as hemothorax-9 cases, pneumothorax -1 , lung contusions- 1 case, rib fractures -8 cases, vertebral fractures- 1 case and pelvic fractures -6 cases, thereby providing additional information in guiding the initial mode of management of such patients.

\section{DISCUSSION}

The most prevalent cause of death is trauma. Following the extremities and head, the next most likely region to be injured is abdomen and surgery is required in $25 \%$ of patients. Penetrating trauma is easily diagnosed whereas blunt trauma because of lack of clinical signs is not easily diagnosed.

In most studies, the major mechanisms of injury are road traffic accidents (RTA), followed by fall34. In the present study RTA accounted for $77.4 \%$ of in injuries and $19.3 \%$ of patients sustained injuries due to fall from height. The prevalence of trauma was more in males (77\%) as reported in earlier studies52. The complete role of plain radiograph is limited in the evaluation of blunt trauma. The chest $\mathrm{X}$ ray can be used for the diagnosis of pneumoperitoneum and diaphragmatic rupture in blunt trauma patients. The pelvic or chest radiograph can demonstrate thoracic and lumbar spine fractures. The presence of transverse fractures of the vertebral bodies (ie, Chance fractures) suggests a higher likelihood of blunt injuries to the bowel. In addition, free intraperitoneal air, or trapped retroperitoneal air from duodenal perforation, may be seen.

Of the total 40 patients, free intraperitoneal air under the diaphragm was seen in 2 patients on abdominal radiograph. Pneumoperitoneum does not always indicate bowel rupture and can be observed in patients with pneumomediastinum or pneumothorax and in patients on mechanical ventilation. The sensitivity of $33 \%$ in our study is similar to that found by Stapakis JC et al. ${ }^{17}$ in detecting free air on abdominal radiograph in comparison with CT. Organ injury can be easily diagnosed by abdominal ultrasound as well as the presence of free intra-abdominal fluid, which could be blood or intestinal secretions. US is cheap, easily available, portable, non-invasive, with no radiation and is done in the emergency unit at the same time with resuscitation methods. Focused abdominal sonography for trauma (FAST) is a fast examination method that could demonstrate intraperitoneal fluid. Radwan et al. ${ }^{18}$ (2006), evaluated the role of FAST and $\mathrm{CT}$ in BAT patients. They suggested that FAST is useful as an initial diagnostic 61 tool to detect free fluid. Out of 40 patients in our study, Ultrasound detected free fluid in 39 patients.

The importance of Computed tomography in the detection 
of blunt trauma lies in its accuracy of detecting injuries that needs exploration and also provides severity of the injury which helps in deciding the management. The rate of negative laparotomy is reduced by avoiding surgical intervention in cases that can be managed conservatively. Though US is sensitive and a widely available preliminary investigative modality, it is inferior in detecting retroperitoneal and bowel injuries, and is operator dependent. Availability of CT is still limited, but it is diagnostically superior to US with its biggest advantage being the ability to assess the severity of trauma and providing a guide to further management. CT can also simultaneously assess other injuries related to trauma.

Haemodynamic instability of the patient is a major deterrent for CT examination, although this forms an indication for surgery in patients with abdominal trauma. Hemodynamic instability, disturbed level of consciousness and presence of other injuries in the skull, chest, pelvic bones or extremities, all explain the need of an accurate and rapid imaging tool to diagnose associated abdominal visceral injuries.

Hemoperitoneum is cited to be the most frequent sign of abdominal injury. Out of the total 40 patients in our study, hemoperitoneum was detected in 39 patients on CT $(96.7 \%)$. Thirty cases were detected on US (75\%). US thus had a sensitivity of $85.3 \%$, specificity of $100 \%$ and an accuracy of $86 \%$ in the detection of hemoperitoneum. Leading source of hemoperitoneum is hepatic trauma. In CT, HU value of more than 30 is more accurate in diagnosis. Due to the time running between scan and the laparotomy (hours) the detection of late hemorrhage is given as false negative.

Splenic injuries account for about $40 \%$ of all intra-abdominal injuries 49. The presence of pulp tissue and poorly developed mesenchymal supporting structure predisposes spleen to injury. In our study spleen was the commonest organ injured with an incidence of $42.5 \% .^{19}$

Liver was the first most frequently injured organ in our study with an incidence of 55\%. 19 cases (47.5\%) of liver injury were detected by US and 55\% detected on CT among all the cases of blunt injury to the abdomen in this study. On US the sensitivity, specificity and accuracy were $84 \%, 99 \%$ and $86 \%$. It has been observed that US is better at diagnosing liver injuries compared to splenic injuries.

The single patient of bladder rupture in our study could be detected on CT. CT showed contrast extravasation from bladder on delayed images. Most authors ${ }^{21}$ have observed that CT was more sensitive than cystography in detection of small amount of contrast extravasation and simply clamping the Foley's catheter before starting the scan may be adequate for all significant injuries. In the single patient in our study with a $1.5 \mathrm{~cm}$ sagittal laceration in the posterior wall, extravasation was seen through the small laceration (grade II) from the collapsed bladder with clamped Foley's catheter. Different workers have tried to predict the need for surgical management based on the grades of organ injury. In our study there was a relatively good correlation of CT grades of splenic injuries with the need for surgical or conservative management. Of total 2 patients with grade I injury one patient is managed conservatively and other required surgery because of multi- organ injury and all the patients with grade II,III and IV injuries required surgery
In a study reported by Liu et al. ${ }^{22} \mathrm{CT}$ missed only one of the seven bowel injuries where as many as three were missed on US. They concluded that in the context of organ injuries, overall, CT and US had a similar sensitivity, specificity and accuracy but differed mainly in respect of detection of isolated small bowel perforation and retroperitoneal hematomas

The fourth commonest injured organ was the kidney (7.5\%). The US detection of renal injury had a sensitivity, specificity and accuracy of $33.3 \%, 100 \%$ and $60 \%$. The specificity and accuracy are comparable to previous studies done by Malliketal. ${ }^{20}$ who reported a sensitivity of $67 \%, 100 \%$ and 100 . In the present study, the age group which was mostly affected was between 16-45 years. Probability for this age group is due to increased outdoor activity and age $>50$ years are less active. Males being more active outdoors are more prone to accidents than females. In our study male predominance (77.5\%) was found.

\section{CONCLUSION}

US thus had an overall sensitivity of $85.3 \%$, specificity of $100 \%$ and accuracy of $86 \%$. CT was positive (either for intra-abdominal free fluid or organ injury or both) in all the patients and thus showed an overall sensitivity, specificity and accuracy of $100 \%$.

Finally, the following conclusions were drawn:-

- US is a valuable initial modality for evaluation of patients with abdominal trauma.

- $\quad$ CT is required in most US positive patients to delineate the exact extent of injury and to exclude any other significant injuries. Also, in a small but significant group CT may change the management approach.

- Symptomatic patients should have a CT even if US examination is negative.

- Inadequate US evaluation in the presence of gaseous distension or overlying surgical emphysema should be followed by CT.

- US or CT quantification of hemoperitoneum or grading of injury does not always dictate whether the management should be conservative or surgical. However they reflect the severity of injury and injuries of severe grade with large free fluid are more likely to require a laparotomy.

\section{REFERENCES}

1. Raptopoulos.V. Abdominal trauma; emphasis on Computed Tomography. Radiol Clin North Am 1994;32(1):969-987.

2. Shiryazdi M, Modir A. Study of the diagnostic value of ultra sonography in blunt abdominal traumas. Pak J Med 2005;44:3(5):130-32.

3. Brown CK, Dunn KA, Wilson K. Diagnostic evaluation of patients with blunt abdominal trauma: a decision analysis. Acad Emerg Med 2000;7(4):385-96.

4. Deitch EiA. 'Commentary' on unnecessary laparotomies for trauma: a prospective study of morbidity. The year book of Surgery 1996. St. Louis: Mosby 1996;4(1);106107.

5. Wolfman NT,Bech told RE et al. Blunt upper abdominal trauma: evaluation by CT. AJR 1992; 158(2):493-501.

6. Fabian TC, Croce MA. Abdominal trauma, including indications for celiotomy. In Feliciano DV, Moore EE, 
Mattox KL: Trauma Stamford, Connecticut: Appleton \& Lange 3rd Ed. 1996: 441-459.

7. Kristensen JK, Buemann B, Kuhl E. Ultrasonic scanning in the diagnosis of splenic haematomas. Acta Chir Scand 1971; 137(6): 653-57.

8. Goletti O, Ghiselli G, et al. The role of ultrasonography in blunt abdominal trauma: J Trauma 1994; 36(4):178181

9. Bode PJ, Niezen RA, et al. Abdominal US as a reliable indicator for conclusive laparotomy in blunt abdominal trauma. J Trauma 1993; 34(1):27-31.

10. Boulanger BR, Brenneman FD, et al. A prospective study of emergent abdominal sonography after blunt trauma. J Trauma 1995; 39(5):325-300.

11. Rozycki GS, Abdominal US in trauma. Surg Clin North Am 1995; 75(1):175-191. 75

12. Goletti $\mathrm{O}$, Ghisell $\mathrm{G}$ et al. The role of Ultrasound in Blunt abdominal trauma J Trauma 1994;36(3):178-181.

13. Mc Williams RG, Johnson MI, et al. Case report: the 'veiled right kidney sign'-an US finding in retroperitoneal perforation of the duodenum.Br J Radiol1996;69(2):1061-1063.

14. Mc Gahan JP, Rose J, et al.- Use of US in acute abdominal trauma. J Ultrasound Med 1997; 16(4):653662.

15. Sirlin CB, Brown MA. Blunt abdominal traumaClinical value of negative screening US. Radiology 2004; 230(1):661-668.

16. Mohamed M Radwan, Fikri M Abu-Zidan: FAST and CT scanning in Blunt abdominal trauma. Surgeons perspective. African Health sciences 2006(3):187-190.

17. Boone DC, Federle M, Evolution of management of hepatic trauma: Identification of patterns of injury $\mathrm{J}$ Trauma1995;39(5);344-350.

18. Moore EE, Cogbill TH,et al. Organ injury scaling. SurgClin North Am 1995; 75(1): 293- 303.

19. Joseph K.T. Lee, Stuart S. Sagel-Computed tomography with MRI correlation 4th Ed. 1444-1446.

20. Khsitish M, Sushma. Comparative evaluation of US and $\mathrm{CT}$ in the evaluation of abdominal trauma.Indian J RadiolImag-2000;10(1);237-243.

21. Goldstein AS, Sclafani SJA, et al. The diagnostic superiority of CT. J Trauma 1985; 25(5):938-946.

22. Liu M, Lee $\mathrm{CH}$, Prospective comparision of diagnostic peritoneal lavage, CT scanning and US for the diagnosis of blunt abdominal trauma. J Trauma 1993;35(3):267270.

Source of Support: Nil; Conflict of Interest: None

Submitted: 08-04-2020; Accepted: 29-04-2020; Published online: 31-05-2020 